\title{
WALL IMPEDANCES FOR LOW AND MODERATE ENERGIES
}

\author{
A. Burov, Budker INP, 630090, Novosibirsk, Russia
}

\begin{abstract}
Properties of the wall impedance of a storage ring are studied for a low relativism, $\gamma-1<1$. Both broad and narrow band impedances are shown to be damped at low energies, the damping factors are found. Coherent motion of a coasting beam is discussed; Landau decrements are calculated.
\end{abstract}

\section{INTRODUCTION}

The impedance of a vacuum chamber [1] depends on a beam velocity. This dependence vanishes in an ultrarelativistic limit, where all the ®elds excited by a point charge lag behind it, which is referred to as the causality principle for wake ®elds, see e. g. [2]. This causality principle does not work for low and moderate energies, where the relativistic factor $\gamma \simeq 1$ : the Coulomb Beld is not here a $\delta$-function of a longitudinal coordinate, but smoothly increase and decrease during the time $\tau \simeq r / \gamma v$, where $r$ is an impact parameter. It follows that a broad band wall impedance $Z(\omega)$ is exponentially depressed at frequencies $\omega \geq \gamma v / b$, where $\mathrm{b}$ is an aperture radius. A pure space charge impedance is the only remaining at these frequencies, but it does not lead to instabilities of itself. A narrow band impedance is shown to be depressed to a lesser extent, then the broad band one, due to a rather sharp boundary of the eigen $® e l d$ at the entrance, $\Delta z=b / 2.4$.

A coherent motion of coasting beam is discussed; the Landau damping of both longitudinal and transverse oscillations is lost when a space charge of the beam separates coherent and incoherent frequencies [6], [9], [10]. Longitudinal and transverse Landau decrements are asymptotically calculated here for a thermal equilibrium.

\section{IMPEDANCE DAMPING FACTORS}

\section{A. Wall Resistivity}

Assuming the $® e l d$ dependence on the longitudinal coordinate and time as $e^{i k(z-v t)}$, Maxwell's equations reduce to the Poisson equation for a longitudinal electric ®eld $E_{z}$ excited by a charge linear density perturbation. Applying Leontovich boundary condition at the resistive wall surface, [3], $E_{z}(b)=$ $\sqrt{-i k v /(4 \pi \sigma)} B_{\theta}$, the electric @eld $E_{z}$ in a perfectly conducting tube and its perturbation $\tilde{E}_{z}$ due to a ®nite walls conductivity $\sigma$ [4] can be found.

The ®elds $E_{z}, \tilde{E}_{z}$ and the current perturbation $J$ are connected by corresponding impedances, $E_{z} C=-Z \| J, \tilde{E}_{z} C=$ $-\tilde{Z} \| J$, where $C$ is the ring circumference, which gives:

$$
\frac{Z^{\|}(k v)}{C}=\frac{2 i k L}{\gamma^{2} v} ; \quad \frac{\tilde{Z} \|(k v)}{C}=\frac{1-i k /|k|}{2 \pi b \delta \sigma} f(\kappa)
$$

where $\delta=c / \sqrt{2 \pi \sigma k v}$ is the skin depth, $L=\ln \left(r_{\max } / a\right)+$ $1 / 2$ is the logarithmic factor with $r_{\max }=\min (b, 1 / k), a$ is the beam radius. Here a high frequency damping factor $f(\kappa)$ has been introduced:

$f(\kappa)=\kappa \frac{K_{1}(\kappa) I_{0}(\kappa)-K_{0}(\kappa) I_{1}(\kappa)}{I_{0}^{2}(\kappa)}= \begin{cases}1, & \text { if } \kappa \ll 1 \\ \pi e^{-2 \kappa}, & \text { if } \kappa \gg 1\end{cases}$

$K_{m}(\kappa), I_{m}(\kappa)$ are modi®ed Bessel functions.

The transverce resistive-wall impedance $\tilde{Z}^{\perp}$ is found by the similar way; for any relativism $\tilde{Z}^{\perp}=2 \tilde{Z} \| /\left(k b^{2}\right)$.

The real part of $\tilde{Z} \|(\kappa)$ achieves its maximum at the dimensionless wavenumber $\kappa=0.43$,

$$
\operatorname{Re} \tilde{Z} \|_{\text {max }}=0.24 Z_{0}(R / b) \sqrt{\gamma v /(2 \pi \sigma b)},
$$

$Z_{0}=4 \pi / c=377 \Omega$. Assuming $\beta=0.4, \sigma=1.3$. $10^{16} \mathrm{~s}^{-1}, R=10 \mathrm{~m}, \quad=5 \mathrm{~cm}$, it gives $\operatorname{Re} \tilde{Z}_{\max }^{\|}=3 \Omega$.

\section{B. Broad Band Impedances}

The resistive wall impedance $\tilde{Z} \|$ (Eq.1) can be represented in terms of its ultra relativistic value $\tilde{Z}_{u r}^{\|}$and the damping factor $f(\kappa)($ Eq. 2$)$ :

$$
\tilde{Z}^{\|}(\omega)=\tilde{Z}_{u r}^{\|} f(\kappa), \quad \kappa=\omega b /(\gamma v) .
$$

The factor $f(\kappa)$ re $^{-}$ects the strong decrease of the incident $® e l d$ energy -ux at the distance $r=b$ as $\sim \exp (-2 k b / \gamma)$. The incident $®$ eld of the beam, damped near the walls as $f^{1 / 2} \sim$ $\exp (-k b / \gamma)$, produces proportionally to itself a perturbation of the surface current density. The energy loss which is a product of the $® e l d \tilde{E}_{z}$ and the current induced, is damped quadratically, as $f^{1 / 2} \cdot f^{1 / 2}=f \simeq \pi \exp (-2 k b / \gamma)$. The energy loss is proportional to the real part of the impedance, so the last one is damped in the same way.

Thus, Eq.(3) follows from the symple physical consideration, which is the same for all the types of the wall imperfections. The only importance is that $®$ eld perturbations introduced by these imperfections are small, which practically can be applied to all broad band wall impedances.

The real part of the broad band impedance has non-zero value only above the low frequency cut-off: $\omega \geq \omega_{c}$. For the circular cross-section of the vacuum chamber $\omega_{c}=2.4 \mathrm{c} / \mathrm{b}$. Taking into account that $\omega=k v$, the damping factor $f$ near the lowest possible frequency (cut-off) occurs to be:

$$
f \simeq \pi \exp (-4.8 /(\gamma \beta))
$$

So, the broad band impedance of wall imperfections (discontinuities, shallow cavities, irises, etc.) cannot play any role for insuf®ciently relativistic particles. Even for $\beta=0.4$, the damping factor $f=2 \cdot 10^{-5}$, which makes the broad band impedance completely negligible.

For a transverse broad band impedance, $\tilde{Z}^{\perp}$ the low energy suppression is actual even to the grater extent. The cut-off frequency for unsymmetrical modes of the vacuum pipe $\omega_{c}^{\perp}$ is signi®cantly higher, than for symmetrical ones, $\omega_{c}^{\perp}=3.8 \mathrm{c} / \mathrm{b}$ for 
the circular pipe, which gives the folowing transverse broad band damping factor:

$$
\tilde{Z}^{\perp}(\omega)=\tilde{Z}_{u r}^{\perp}(\omega) f_{\perp}(\kappa), \quad f_{\perp}(\kappa) \simeq \exp (-7.6 /(\gamma \beta)) .
$$

\section{Narrow Band Impedances}

Another possibility for energy loss and for instabilities is connected with the radiation in low frequency $\left(\omega<\omega_{c}\right)$ modes of some elements of the vacuum chamber. At the resonance, the impedance is pure real, it achieves here its maximum, the shunt impedance $R_{s}(v)$. The velocity dependence of the shunt impedance is determined by a transite time factor $T(v)$ (see, e. g. [5]):

$$
R_{s}(v)=R_{s}(c)\left|\frac{T(v)}{T(c)}\right|^{2}, \quad T(v)=\int_{-\infty}^{\infty} E(z) e^{-i \omega z / v)} d z,
$$

where $E(z)$ is an arbitrary normalized eigen ${ }^{\circledR}$ eld distribution. It follows the estimation:

$$
\frac{R_{s}(v)}{R_{s}(c)}=[\beta \sin (\omega g /(2 v)) / \sin (\omega g /(2 c))]^{2}\left\{\frac{\delta \psi}{\sinh (\delta \psi)}\right\}^{2}
$$

where $\psi=\omega g / v, g$ is a gap length, $\delta \psi=\pi \omega \Delta z / v$, longitudinal variation of the eigen $® e l d$ in the gap assumed to be $\ll \omega / v$. The $\{\ldots\}$ - factor $\mathrm{re}^{-}$ects an in-uence of a width of the (Beld boundary $\Delta z=b / 2.4$ at the entrance and exit of the narrow band element.

\section{COHERENT STABILITY}

\section{A. Longitudinal Oscillations}

The dispersion relation can be found from the kinetic equation, which is the Vlasov equation plus coolingdiffusion Fokker-Planck term [6]. In the reference frame:

$$
\frac{\partial f}{\partial t}+w \frac{\partial f}{\partial z}+\frac{Z_{i} e}{M_{\|}} E \frac{\partial f_{0}}{\partial w}=\frac{\partial}{\partial w}\left(\lambda_{\|} w f+d_{\|} \frac{\partial f}{\partial w}\right),
$$

where $f_{0}$ is the beam phase density, $f$ is its perturbation, $w$ is a deviation of the particle velocity from the beam velocity $v$, $M_{\|}=M_{i}\left(1 / \gamma^{2}-1 / \gamma_{t}^{2}\right)^{-1}$ is a longitudinal mass of an ion of the beam, $M_{i}=A_{i} M_{p}$ is its mass, $A_{i}$ and $Z_{i}$ are the mass and charge numbers, $\lambda_{\|}$is the cooling rate and $d_{\|}$is the diffusion coef $\circledast$ cient. Assuming the oscillations to be mainly determined by the space charge impedance $Z \|$, the corrections introduced by the cooling, temperature (Landau damping) and the impedance $\tilde{Z} \|$ can be found as perturbations. It gives the following dispersion relation for longitudinal coherent modes in a coasting beam [7]:

$$
\begin{aligned}
\Omega(k) & = \pm k u_{\|}\left\{1+\frac{\pi i}{2} \frac{k}{|k|} u_{\|}^{2} f_{0}^{\prime}(\Omega / k)-i \frac{\tilde{Z} \|}{2|Z \||}\right\}-i \frac{\lambda_{\|}}{2} . \\
u_{\|} & =c \sqrt{2 \bar{\rho} r_{0} L\left(\gamma^{-2}-\gamma_{t}^{-2}\right)}, \quad r_{0}=Z_{i}^{2} e^{2} /\left(M_{i} c^{2}\right),
\end{aligned}
$$

According to Eq.(7), instabilities caused by the real part of the impedance $\operatorname{Re} \tilde{Z} \|$ can be avoided due to the Landau damping or due to cooling. In the ${ }^{\circledR r s t}$ case longitudinal temperature of the beam must be suf®ciently high. Assuming the distribution function to be Gaussian, $f_{0}(u)=\left(2 \pi \Delta w^{2}\right)^{-1 / 2} \exp \left(-u^{2} / 2 \Delta w^{2}\right)$, the stability condition can be expressed as:

$$
\frac{u_{\|}^{2}}{\Delta w^{2}} \leq 2 \ln \left(\sqrt{\frac{\pi}{2}} \frac{u_{\|}^{3}}{\Delta w^{3}} \frac{|Z \||}{\operatorname{Re} \tilde{Z} \|}\right)
$$

The factor $\left|Z^{\|}\right| / \operatorname{Re} \tilde{Z}^{\|}$is usually pretty large. Therefore the stability condition (8) is almost independent on the impedance. In terms of a temperature it can be expressed as:

$$
T^{\|} \geq T_{t h}^{\|}(\mathrm{K})=0.02 \frac{I(\mu \mathrm{A}) Z_{i} L}{\beta\left(1-\gamma^{2} / \gamma_{t}^{2}\right)} f_{Z}
$$

where the factor $f_{Z} \approx 1 \mathrm{re}^{-}$ects the weak logarithmic dependence on the impedance. For instance, longitudinal oscillations in a $1 \mu \mathrm{A}$ beam of $\mathrm{Li}_{7}^{+1} \quad$ [8] with $\beta=0.06, L=4$, will be Landau-damped if its longitudinal temperature $T^{\|} \geq T_{t h}^{\|}=1.6$ $\mathrm{K}$. For $1 \mathrm{~mA}$ beam of $\mathrm{C}_{12}^{+6}$ at TSR with $\beta=0.041$ the threshold temperature calculated from (9) with $L=5, f_{Z}=1.5$ is: $T_{t h}^{\|}=2 \cdot 10^{4} \mathrm{~K}$, which is rather close to the experimental value of $T^{\|}=3 \cdot 10^{4} \mathrm{~K}$.

This limit can be removed if the cooling rate is high enough:

$$
\lambda_{\|}>2 \Lambda_{k}=\frac{u_{\|} \beta \operatorname{Re} \tilde{Z}_{k}^{\|}}{L R Z_{0}}
$$

If this is satis®ed for all the wavenumbers $k=n / R$, the oscillations are stable even without Landau damping. For a given $\lambda_{\|}$ the restriction (10) can be treated as a safe condition imposed on the impedance $\tilde{Z} \|$. Assuming $\lambda_{\|}=10 s^{-1}$, for the $\mathrm{Li}_{7}^{+1}$ beam it gives: $\operatorname{Re} \tilde{Z} \|<10 \mathrm{~K} \Omega$.

\section{B. Transverse Oscillations}

Transverse oscilations of ions of a coasting beam can be described by the following equation:

$\ddot{x}+\omega_{b}^{2} x-\omega_{s c}^{2}(x-\bar{x})=\left(\bar{\rho} r_{0} c^{2} / \gamma\right) \int W_{\perp}\left(s-s^{\prime}\right) \bar{x}\left(s^{\prime}\right) d s^{\prime}-\lambda_{\perp} \dot{x}$,

where $x, \bar{x}$ are transverse coordinates of an individual ion and a center of mass of the beam, $\dot{x}=d x / d t=\left(-i \Omega+i n \omega_{0} \eta w / v\right) x$ is a transverse velocity of the ion, $\eta=\gamma^{-2}-\gamma_{t}^{-2}, w$ is a deviation of a longitudinal velocity from its average value $v$, $\omega_{b}=\omega_{b 0}+\xi \omega_{0} w / v$ is a betatron frequency with a chromaticity accounted, $\bar{\rho}$ is a linear density of the beam, $\omega_{s c}^{2}$ describes a space charge defocusing, $W_{\perp}(s)$ is a transverse wake function, $\lambda_{\perp}$ is a transverce cooling rate. From here, a dispersion equation follows:

$$
1=\int \frac{d I d w f_{\perp 0}(I) f_{\| 0}(w)\left(\omega_{s c}^{2}(I)+\omega_{c}^{2}\right)}{\left(\Omega-n \eta \omega_{0} \frac{w}{v}+i \frac{\lambda_{\perp}}{2}\right)^{2}-\left(\omega_{b 0}+\omega_{0} \xi \frac{w}{v}\right)^{2}+\omega_{s c}^{2}(I)},
$$

where $\bar{\rho}=N / C$ is a linear density of the beam. 
where $\omega_{c}^{2}=2 i \bar{\rho} r_{0} c \omega_{0} \gamma^{-1}\left(Z^{\perp} / Z_{0}\right), I$ is a transverse action, $f_{\perp 0}(I), f_{\| 0}(w)$ are unperturbed distribution functions, $\int d I d w f_{\perp 0}(I) f_{\| 0}(w)=1$. For small Landau damping and cooling corrections, the equation (12) can be solved:

$$
\begin{aligned}
& \Omega=-\left(\omega_{b 0} \uparrow \Delta_{c}\right) n /|n|-i \lambda_{\perp} / 2-i \delta_{\perp} \\
& \Delta \Omega_{c}=i \frac{\bar{\rho} r_{0} c}{\gamma Q_{b}} \frac{Z^{\perp}}{Z_{0}}, \quad Q_{b}=\frac{\omega_{b 0}}{\omega_{0}} \\
& \delta_{\perp}=\pi \Delta \omega_{L} \int d I d w f_{\perp 0}(I) f_{\| 0}(w) \Delta \omega_{s c}(I) . \\
& \cdot \delta\left(\Delta \omega_{s c}(I)+\operatorname{Re} \Delta \Omega_{c}-\xi \omega_{0} w / v+|n| \eta \omega_{0} w / v\right) .
\end{aligned}
$$

Here $\Delta \omega_{s c}(I)=\omega_{s c}^{2}(I) /\left(2 \omega_{b 0}\right)$ is an incoherent space charge betatron shift for ions with the action $I, \Delta \omega_{L}=\Delta Q_{L} \omega_{0}=$ $\Delta \omega_{s c}(0)$ is the Laslett shift.

The Landau damping term $\delta_{\perp}$ is seen to be lost when the incoherent and coherent frequencies are separated more than a width of the distribution. Typically, at low relativism $\Delta \omega_{L} \gg$ $\Delta \Omega_{c}$, which follows the stability threshold for the Laslett shift [9], [10]. Actually, the situation is similar to the logitudinal case, where the space charge separates velocities of particles and waves, also causing the threshold to be almost independent on the ring impedance (Eq.9).

The Landau decrement $\delta_{\perp}$ can be asymptotically calculated from Eq.(13) for a thermal equilibrium, with some longitudinal and transverse temperatures $T_{\|}=M \Delta w^{2}, T_{\perp}$ :

$$
\delta_{\perp}=\pi \sqrt{\frac{2}{3}} \Delta \omega_{L} p^{2 / 3} \exp \left(-3 p^{2 / 3}\right), p=\frac{\Delta Q_{L}}{|-\xi+| n|\eta|} \frac{v}{\Delta w} .
$$

From here, the stability condition for the Landau suppression of the instability can be presented as:

$$
\Delta Q_{L} \leq \Delta Q_{t h} \approx 0.2|-\xi+| n|\eta| \frac{\Delta w}{v} \ln ^{3 / 2}\left(\frac{5 \Delta \omega_{L}}{\Lambda_{n}}\right)
$$

where $\Lambda_{n}=\operatorname{Im} \Delta \Omega_{c}$. The limit value for the Laslett tune shift is determined by the mode with the minimum longitudinal number $n$, which has the increment higher than cooling decrement: $\Lambda_{n}>\lambda_{\perp} / 2$. Due to a large argument of the logarithm $\left(\sim 10^{5} \div 10^{7}\right)$, the result is almost unsensitive on it; the logarithmic factor itself is a large constant: $\ln ^{3 / 2}(\ldots) \approx 50 \div 60$. Assuming the cooling rate to be smaller than long wave $(n \leq|\xi|)$ increments, the threshold Laslett shift is found:

$$
\Delta Q_{t h} \approx 10|\xi| \Delta w / v .
$$

Taking as an example typical for TSR at the threshold $\Delta w / v=$ $10^{-3}$ and $|\xi|=5$, it gives $\Delta Q_{t h}=0.05$, which is close to the observed value [11]. The restriction on the Laslett tune shift (15) is removed for suf®ciently low currents, with the cooling rate higher then coherent increments: $\lambda_{\perp}>2 \Lambda$. For the mentioned Li beam, with $\lambda_{\perp}=1 \mathrm{~s}^{-1}$, it gives the restriction for the impedance: $\operatorname{Re} Z^{\perp}<15 M \Omega / m$.

\section{CONCLUSIONS}

The concept of the storage ring impedance, developed mainly for ultrarelativistic beams cannot be at once applied to low and moderate energy cases. The reason is that the causality principle for wake ®elds generally is not valid here, which cause an exponential damping of impedances above a certain threshold. The approach suggested gives the possibility to $®$ nd the wall impedance for an arbitrary energy if its ultrarelativistic value is known. At low energies, $\beta \leq 0.5 \div 0.8$ the broad-band wall impedance is exponentially damped and too weak to cause instabilities of a cooled beam. A narrow-band impedance could be dangerous if its eigenfrequency $\omega_{r}$ is rather low: $\omega_{r} b / v \leq 1$.

Space charge of a beam switches-off the Landau damping, above certain thresholdes, for both longitudinal and transverse oscillations. For typical cooling rates, low current $(\mu \mathrm{A})$ beams could be cooled below the thresholds.

\section{ACKNOWLEDGMENTS}

The report has been done in the framework of the CRYSTAL project. The author is particularly grateful to $\mathrm{L}$. Tecchio, N. S. Dikansky, A. Aleksandrov, G. Bisof ${ }^{\circledR}$, S. Gustafsson, G. Lamanna, A. Lombardi and A. V. Novokhatski for discussions and information.

\section{References}

[1] A. Sessler and V. Vaccaro, CERN Report ISR-RF/67-2 (1967).

[2] A. W. Chao Physics of Collective Beam Instabilities in High Energy Accelerators, Wiley, N.- Y. 1993.

[3] J. D. Jackson. Classical Electrodynamics, Wiley, N.- Y. and London, 1962.

[4] P. L. Morton, V. K. Neil, and A. M. Sessler, J. Appl. Phys. 37, 3875 (1966).

[5] L. Palumbo, V. Vaccaro and M. Zobov Wake Fields and Impedance, LNF-INFN Report 94/041, 1994

[6] N. S. Dikansky, D. V. Pestrikov. The Physics of Intense Beams and Storage Rings, AIP, N.- Y. 1994.

[7] A. Burov, in Proc. of the Workshop on Beam Cooling and Related Topics, p.230, 1994.

[8] L. Tecchio et al. CRYSTAL. A Storage Ring for Crystalline Beams. LNL-INFN Int. Rep., 1994.

[9] L. J. Laslett, A. M. Sessler, D. Mタ̀nl, NIM, 121, p. 517.

[10] B. Zotter, F. Saherer, CERN Report 77-13, p.175, 1977

[11] M. Grieser, in Coll. of Transp. of Disc. on Cold Stored Ion Beams, Heidelberg, July 7-8, 1994.

[12] A. Burov, Electron Cooler Impedances, these Proceedings. 